\section{Late presentation of delayed hemothorax after blunt chest trauma in a patient with impaired liver function}

\author{
Georg Authried, ${ }^{1,2}$ \\ Ane Sogaard Teisner, ${ }^{1,3}$ Michael Stenger ${ }^{4}$ \\ 'Department of Emergency Medicine, \\ Odense University Hospital, Odense; \\ 2Department of Dermatology and Allergy, \\ Odense University Hospital, Odense; \\ ${ }^{3}$ Department of Medical Gastrointestinal \\ Diseases, Odense University Hospital, \\ Odense; ${ }^{4}$ Department of Cardiothoracic \\ Surgery, Odense University Hospital, \\ Odense, Denmark
}

\begin{abstract}
Delayed hemothorax (DHTX) after blunt chest trauma (BCT) is fairly uncommon but nevertheless associated with a substantial morbidity. DTHX is often related to several or displaced rib fractures and usually presented within the first few days or weeks after the chest trauma. We present a case with an unusual late presentation of DTHX $4 \frac{1}{2}$ months following a BCT with several non-displaced rib fractures after falling down a staircase. Furthermore, we argue that patients with impaired liver function may have an increased risk of bleeding complications after BCT with associated rib fractures and should be considered for more intensive follow-up.
\end{abstract}

\section{Introduction}

The majority of thoracic traumas are caused by blunt injuries. Hemothorax following blunt chest trauma (BCT) may be associated with significant morbidity and mortality depending on the extent of injury to the underlying bone, lung or vascular structures. In adults, especially the presence and the number of rib fractures are predictors for hemothorax, severity and mortality rates. ${ }^{1,2}$ Delayed hemothorax (DHTX) is a fairly uncommon complication after BCT and rarely reported but also closely related to multiple or displaced rib fractures and significant morbidity. ${ }^{3,4}$ In previous reports DTHX has been recognized within a few days and maximum 4 weeks after the recorded BCT. ${ }^{1-5}$ We present a case with unusual late presentation of DTHX $4 \frac{1}{2} 2$ months following a BCT with several non-displaced rib fractures after falling down a staircase. Furthermore, we argue that patients with an increased risk of bleeding complications, e.g. due to impaired liver function may need closer follow-up after BCT with multiple or displaced rib fractures.

\section{Case Report}

A 63-year-old male with a history of alcohol abuse, hypertension, and psoriasis vulgaris was admitted to the Emergency Department with nausea, shortness of breath and signs of premature circulatory shock. Four and a half months earlier the patient had suffered a significant BCT to the right hemithorax after falling down a staircase. Three non-displaced rib fractures and no pneumo- or hemothorax were at that point identified clinically and by chest X-ray (Figure 1). He received epidural analgesic treatment and was discharged after one week of recovery.

Upon the current admission, the patient during the past couple of weeks experienced increasing fatigue, vertigo, nausea, and breathing problems. No complaints or objective signs indicating a new or resent chest trauma. Vital signs were: pulse 147 beats per minute, blood pressure $96 / 74 \mathrm{mmHg}$, peripheral saturation $99 \%$ with $2 \mathrm{~L}$ of oxygen, respiratory frequency 19 per minute, temperature $36.3^{\circ} \mathrm{C}$, Glascow Coma Scale 15 .

Arterial blood gas analysis was overall normal except from carbon dioxide partial pressure $4.0 \mathrm{kPa}$ (ref.: 4.7-6.4) and lactate 9.0 $\mathrm{mmol} / \mathrm{L}$ (ref.: 0.5-2.5). Thus, mild hyperventilation and elevated lactate due to tissue hypoperfusion secondary to hypotension were registered.

Additional lab results: hemoglobin 7.2 $\mathrm{mmol} / \mathrm{L}$ (ref.: 8.3-10.5), albumin $33 \mathrm{~g} / \mathrm{L}$ (ref.: 36-48) coagulation factors (II, VII, X) $0.51 \mathrm{U} / \mathrm{L}$ (U/L) (ref.: 0.70-1.30), international normalized ratio 1.3 , lactate dehydrogenase $251 \mathrm{U} / \mathrm{L}$ (ref.: 105-205), alanine transaminase $57 \mathrm{U} / \mathrm{L}$ (ref.: 10-70), alkaline phosphatase $190 \mathrm{U} / \mathrm{L}$ (ref.: 35-105), bilirubin $36 \mu \mathrm{mol} / \mathrm{L}$ (ref.: 5-25), gamma glutamyl transferase 904 U/L (ref.: 15115).

After rehydration with $2 \mathrm{~L}$ of isotonic saline vital signs were partially normalized and lactate was reduced to $3.7 \mathrm{mmol} / \mathrm{L}$. Auscultation and percussion of the lungs revealed attenuated sounds on the right side. An acute chest $\mathrm{x}$ ray and the following computed tomography scan showed massive pleural effusion on the right side with compression atelectasis of the lung and callus formation on four non-displaced rib factures (costae 5-8) incurred 41/2 months ago (Figure 2A-C). He was treated with a chest tube and $1350 \mathrm{~mL}$ of old, dark and partially clotted blood was immediately evacuated from the pleural cavity (Figure 2D). No signs of fresh intra-pleural bleeding were observed.
Correspondence: Michael Stenger, Department of Cardiothoracic Surgery, Odense University Hospital, Sdr. Boulevard 29, DK-5000 Odense, Denmark.

Tel: +45.61691711 - Fax +45.65916539.

E-mail: michael.stenger@rsyd.dk

Key words: Delayed hemothorax; Blunt chest trauma; Impaired liver function.

Received for publication: 7 December 2015.

Revision received: 30 June 2016.

Accepted for publication: 5 August 2016.

This work is licensed under a Creative Commons Attribution NonCommercial 4.0 License (CC BYNC 4.0).

(C) Copyright G. Authried et al., 2016

Licensee PAGEPress, Italy

Chest Disease Reports 2016; 4:5673

doi:10.4081/cdr.2016.5673

\section{Outcome and follow-up}

The patient was discharged after 6 days of hospitalization. Within the following 4 months he was re-admitted twice with shortness of breath and drained for recurrent pleural effusions on the right side.

\section{Discussion}

Ross et $a l .{ }^{5}$ were the first to publish two cases of DTHX after BCT with rib fractures but only a few authors have published larger retrospective series. Sharma et $a .^{4}$ reported a series of 167 patients with hemothorax following BCT over an 8-year period. 160 were diagnosed as acute hemothorax and only 7 cases (4.2\%) developed hemothorax with a delay of 22 hours to 16 days. All patients with DTHX were males above the age of 40 and with multiple rib fractures with displacement in $71 \%$ of the cases. Simon et al. ${ }^{3}$ reported 36 cases of hemothorax after BCT over a three-year period. 12 patients (33\%) presented a DTHX with a delay of 18 hours to 6 days. Eleven of the 12 cases with DTHX had multiple or solitary displaced rib fractures. Finally, McLoughlin et al. ${ }^{2}$ presented a series of 30 cases with hemothorax in 220 patients with rib fractures over a period of $4 \frac{1}{2}$-years. In 16 patients (53\%) it developed with a delay of maximum 28 days, which so far is the longest reported time delay, making the present case very unique. We stress the fact that we have no evidence to believe a new or recent chest trauma had occurred. However, with respect to the current case and previous reports, ${ }^{2-4}$ we note the possibility of bias due to general lack of follow-up and radiological documentation in the inter- 
vening period after the primary BCT until the point of presentation. One may speculate that in the present case the actual time of delay was shorter than $4 \frac{1}{2}$ months since the patient had suffered from increasing fatigue, vertigo, nausea, and breathing problems over the past couple of weeks.

As of identifying patients at risk of developing DTHX, previous reports agree on a strong correlation with multiple and/or displaced rib fractures. $^{2-4}$ Correspondingly, Liman et al. ${ }^{1}$ identified in a series of 1490 patients with BCT that the presence of more than two rib fractures, age $>60$ and a injury severity score of $\geq 16$ are strong predictors for hemothorax, morbidity and mortality.

\section{Conclusions}

The current patient meets the risk factors of multiple rib fractures and age. Furthermore, he did not receive any anti-coagulant therapy,
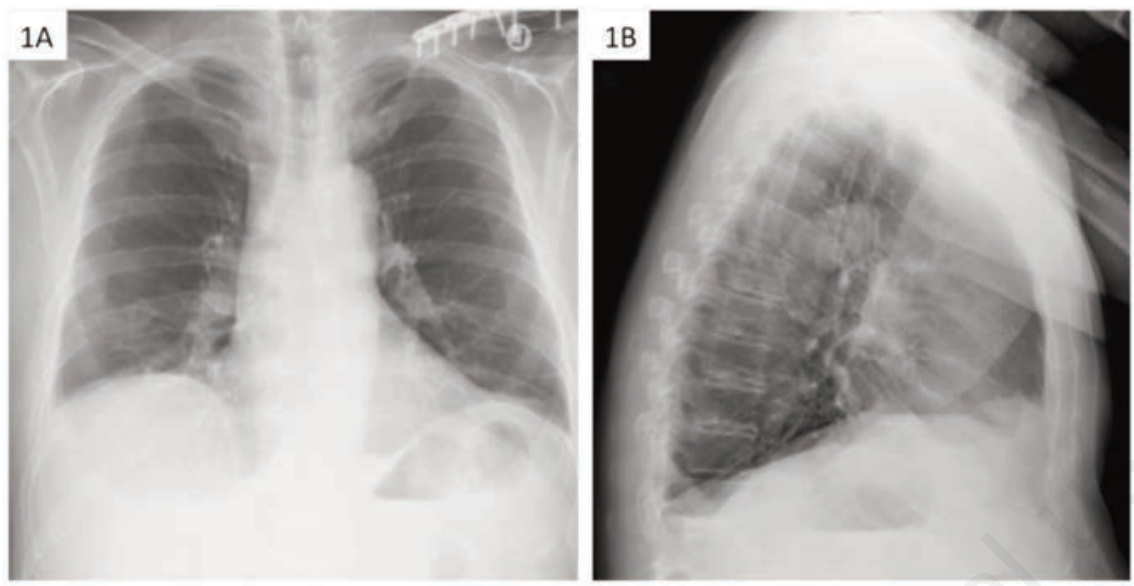

Figure 1. Two chest $\mathrm{X}$-rays [posteroanterior (A) and lateral (B) projection] of a 63-yearold male (case) performed immediately after a blunt chest trauma to the right hemithorax showing several non-displaced rib fractures on the right side and no pneumo- or hemothorax.
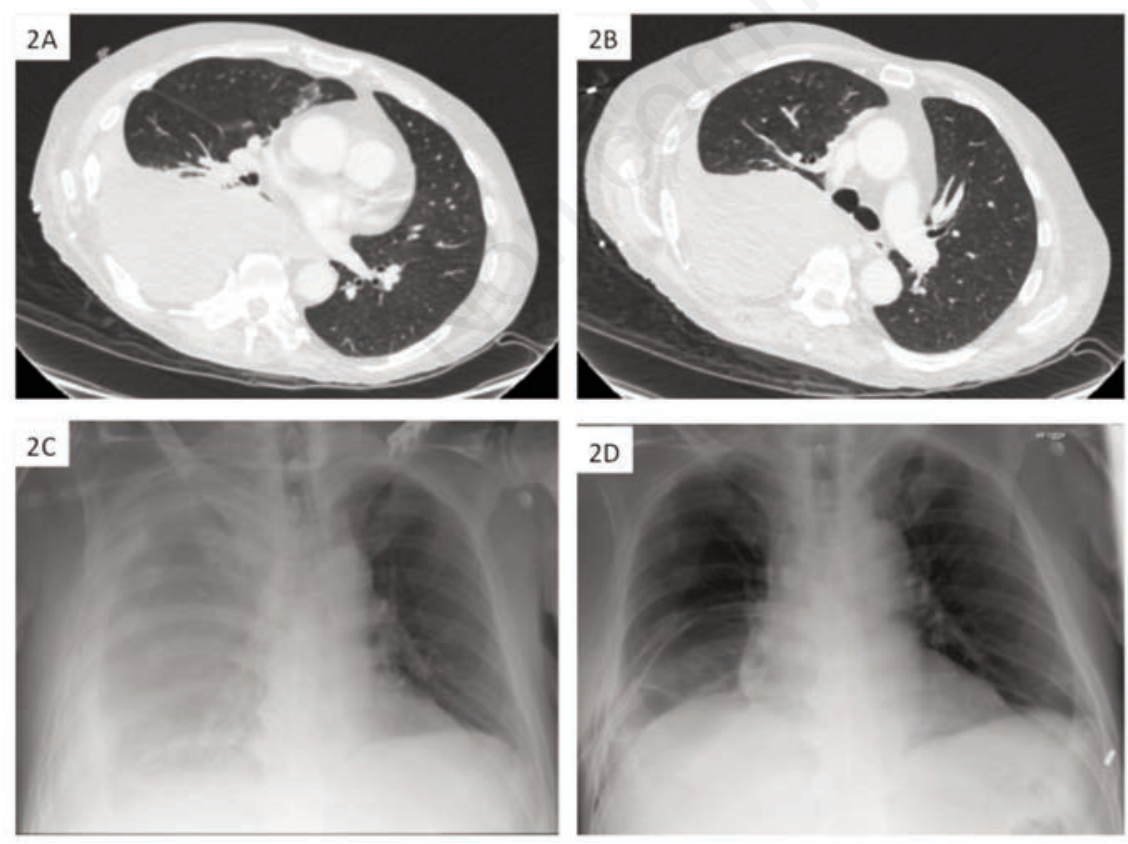

Figure 2. Two transversal computed tomography images (A, B) and two chest X-rays (C, D) of a 63-year-old male (case) performed $4 \frac{112}{2}$ months after the blunt chest trauma to the right hemithorax. Massive pleural effusion on the right side with compression atelectasis of the lung and callus formation rib factures costae (5-8) were identified (A-C). The right pleural cavity was drained by chest tube insertion (D).

but he had a history of alcohol abuse with affected liver enzymes and coagulation factors, which hypothetically over a prolonged period may have caused an on/off-bleeding condition explaining the unusual late presentation. Hence, we argue that patients with impaired liver function due to alcoholic or chronic liver disease should be considered for more thoroughly follow-up after a BCT with associated rib fractures.

\section{References}

1. Liman ST, Kuzucu A, Tastepe AI, et al. Chest injury due to blunt trauma. Eur J Cardio-Thorac 2003;23:374-8.

2. McLoughlin R, Mulcahy R, Kent P, et al. Haemothorax after rib fracture-incidence, timing and prediction. Irish J Med Sci 1987;156:117-9.

3. Simon BJ, Chu Q, Emhoff TA, et al. Delayed hemothorax after blunt thoracic trauma: an uncommon entity with significant morbidity. J Trauma 1998;45:673-6.

4. Sharma OP, Hagler S, Oswanski MF. Prevalence of delayed hemothorax in blunt thoracic trauma. Am Surgeon 2005;71:4816.

5. Ross RM, Cordoba A. Delayed life-threatening hemothorax associated with rib fractures. J Trauma 1986;26:576-8. 